\title{
Supporting Information: Discerning Among All Possible Activation Mechanisms of a Plasmon-Driven Chemical Reaction
}

Rifat Kamarudheen, ${ }^{1,2, \S}$ Guus J. W. Aalbers, ${ }^{1, \S}$ Ruben F. Hamans, ${ }^{1,2,4 \S}$ Leon P. J. Kamp ${ }^{3}$ and Andrea Baldi $i^{1,2,4^{*}}$

${ }^{1}$ DIFFER - Dutch Institute for Fundamental Energy Research, De Zaale 20, 5612 AJ Eindhoven, The Netherlands

${ }^{2}$ Institute for Complex Molecular Systems and ${ }^{3}$ Fluid Dynamics Laboratory, Department of Applied Physics, Eindhoven University of Technology, 5600 MB Eindhoven, The Netherlands ${ }^{4}$ Department of Physics and Astronomy, Vrije Universiteit Amsterdam, De Boelelaan 1081, 1081 HV Amsterdam, The Netherlands

${ }^{\S}$ These authors contributed equally to this work.

*e-mail: a.baldi@vu.nl

\section{Contents}

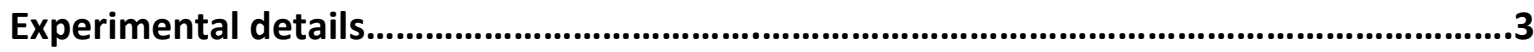

Supplementary S1: Morphological and optical characterization of Au \& Au@Ag core@shell nanorods

Supplementary S2: Modeling photon propagation in our reaction volume

Supplementary S3: Ex situ extinction measurements and morphological characterization

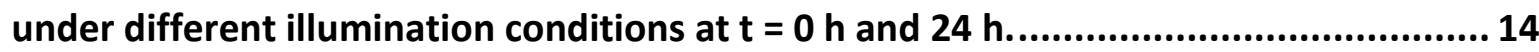

Supplementary S4: Evaluating plasmonic rate enhancement .................................... 16

Supplementary S5: Thermal characterization under laser illumination ......................... 17

Supplementary S6: Quantifying near-field enhancements........................................ 21

Supplementary S7: Temperature calculations in single-particle studies..................... 22

Supplementary S8: Mie theory calculations for the growth of Au@Ag core@shell nanospheres

Supplementary S9: Control experiments to confirm the plasmonic nature of silver shell

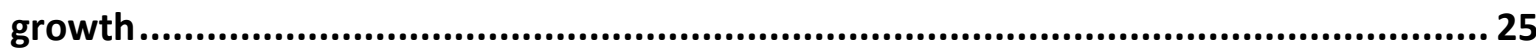

Supplementary S10: Monte Carlo simulations of hot carrier propagation..................... 27

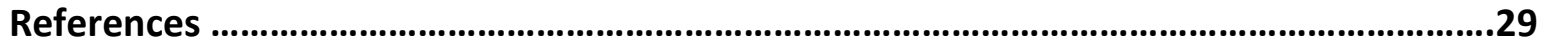




\section{Experimental details}

\section{Materials and methods}

L-Ascorbic acid ( $\geq 99 \%)$, bis(p-sulphonatophenyl)phenylphosphine dihydrate dipotassium salt (97\%), silver nitrate (99.9999\%), cetyltrimethylammonium chloride (CTAC, 25 wt.\% in $\mathrm{H}_{2} \mathrm{O}$ ), gold(III) chloride trihydrate $(\geq 99.9 \%)$, potassium bromide $(\geq 99 \%)$, sodium borohydride (99\%), cetyltrimethylammonium bromide (CTAB, $\geq 98 \%)$ are purchased from Sigma Aldrich. All the solutions are prepared using deionized Milli-Q water $\left(18.2 \mathrm{M} \Omega \mathrm{cm}\right.$ at $\left.25^{\circ} \mathrm{C}\right)$.

\section{Synthesis of gold nanorods}

Gold nanorods are synthesized by adapting a previously reported colloidal seed mediated technique. ${ }^{1}$ Spherical gold seeds are synthesized first by mixing aqueous solutions of $\mathrm{HAuCl}_{4}$ (5 mL, $0.5 \mathrm{mM}$ ) and CTAB $(5 \mathrm{~mL}, 200 \mathrm{mM})$ in a round bottom flask (RBF). Ice-cold $\mathrm{NaBH}_{4}(0.6$ $\mathrm{mL}, 10 \mathrm{mM}$ ) is added to the RBF in one-shot, under vigorous stirring. The solution is aged for $30 \mathrm{~min}$ at room temperature.

To synthesize the nanorods, CTAB (1.8 g) and $\mathrm{KBr}(0.7 \mathrm{~g})$ are dissolved in $50 \mathrm{~mL}$ of warm water in another RBF, followed by cooling the flask to $30{ }^{\circ} \mathrm{C} . \mathrm{AgNO}_{3}(2.4 \mathrm{~mL}, 4 \mathrm{mM}), \mathrm{HAuCl}_{4}$ (50 mL, $1 \mathrm{mM})$, and ascorbic acid $(0.9 \mathrm{~mL}, 64 \mathrm{mM})$ are added to the above solution at intervals of $15 \mathrm{~min}$. The solution is mixed well after each addition step. Finally, the gold seed solution $(0.16 \mathrm{~mL})$ is added to the above mixture and stirred for $30 \mathrm{~s}$, and left undisturbed for $12 \mathrm{~h}$ at $30{ }^{\circ} \mathrm{C}$. The nanorods are washed multiple times in $25 \mathrm{mM}$ CTAC solutions by centrifugation at $8000 \times \mathrm{g}$ for 25 minutes. The washed nanoparticles are finally dispersed in Milli-Q water. 


\section{Synthesis of Au@Ag core@shell nanorods}

Au@Ag core@shell nanorods are synthesized by adapting a previous report. ${ }^{2}$ Gold NRs $(60 \mu \mathrm{L})$ are mixed with water $(960 \mu \mathrm{L})$ to obtain a final optical density of $\sim 0.36$. Aqueous solutions of BSPP $(30 \mu \mathrm{L}, 375 \mathrm{mM}), \mathrm{AgNO}_{3}(30 \mu \mathrm{L}, 150 \mathrm{mM})$, and ascorbic acid $(90 \mu \mathrm{L}, 1.5 \mathrm{M})$ are added sequentially to the above suspension. The suspension is mixed well after the addition of each reactant.

$500 \mu \mathrm{L}$ of the above suspension is transferred in to a $10 \times 4 \mathrm{~mm}$ quartz cuvette with a magnetic stir bar. The cuvette is then placed inside a Quantum Northwest Qpod 2e/MPKIT temperature-controlled cuvette holder at $6{ }^{\circ} \mathrm{C}$, under constant stirring at $300 \mathrm{rpm}$. The temperature of the cuvette holder is maintained using an external water bath.

Depending on the experiment, we either keep this suspension in the dark or under illumination by a $532 \mathrm{~nm}$ or $730 \mathrm{~nm} \mathrm{cw}$ laser. In situ extinction measurements are performed by aligning a halogen white light source to a fiber spectrometer, through the 4 $\mathrm{mm}$ path length of the cuvette.

\section{Synthesis of gold nanospheres}

Gold nanospheres of $66 \mathrm{~nm}$ diameter are synthesized by adapting a previously reported colloidal seed mediated technique. ${ }^{3} \mathrm{HAuCl}_{4}(5 \mathrm{~mL}, 0.5 \mathrm{mM})$ and CTAB $(5 \mathrm{~mL}, 200 \mathrm{mM})$ aqueous solutions are mixed together in an RBF, followed by the addition of $\mathrm{NaBH}_{4}(0.6 \mathrm{~mL}$, $10 \mathrm{mM}$ ) in one-shot. The solution is then immediately stirred for $2 \mathrm{~min}$. The solution is kept undisturbed at $27^{\circ} \mathrm{C}$ for $3 \mathrm{~h}$, to synthesize CTAB capped Au nanoclusters.

In a clean RBF, CTAC (40 mL, $200 \mathrm{mM})$, ascorbic acid $(30 \mathrm{~mL}, 100 \mathrm{mM})$, and the CTAB capped gold nanoclusters $(1 \mathrm{~mL})$ are added in sequence and mixed at $27^{\circ} \mathrm{C} . \mathrm{HAuCl}_{4}(40 \mathrm{~mL}$, $0.5 \mathrm{mM}$ ) is then added to the above mixture in one-shot, under constant stirring for $15 \mathrm{~min}$, 
to form $10 \mathrm{~nm}$ gold nanoseeds. These nanoseeds are initially centrifuged ( $18000 \times \mathrm{g}, 90 \mathrm{~min})$ and redispersed in water. These gold nanoseeds are centrifuged again (18000 $\times$ g, $60 \mathrm{~min})$ and redispersed in a CTAC solution $(1 \mathrm{~mL}, 20 \mathrm{mM})$.

In another RBF, CTAC (40 mL, $100 \mathrm{mM})$, ascorbic acid $(2.6 \mathrm{~mL}, 10 \mathrm{mM})$, and $10 \mathrm{~nm}$ gold nanoseeds $(0.1 \mathrm{~mL})$ are added in sequence and mixed at $27{ }^{\circ} \mathrm{C} . \mathrm{HAuCl}_{4}(40 \mathrm{~mL}, 0.5 \mathrm{mM})$ is then added to the above mixture using a syringe pump at a rate of $40 \mathrm{~mL} / \mathrm{h}$, under constant stirring. The final product is centrifuged thrice $(7200 \times \mathrm{g}, 10 \mathrm{~min})$ in $20 \mathrm{mM}$ CTAC and finally dispersed in Milli-Q water.

\section{Synthesis of individual Au@Ag core@shell nanospheres on a glass substrate}

Single-particle studies on the growth of core@shell nanoparticles are performed inside a glass flow cell. Glass slides and coverslips are first treated with $2 \mathrm{M} \mathrm{KOH}$ at $80^{\circ} \mathrm{C}$ for $20 \mathrm{~min}$, followed by sonication in water, to render their surfaces hydrophilic. A few microliters of a very dilute suspension (optical density $<0.01$ ) of $66 \mathrm{~nm}$ gold nanospheres is drop casted on to the coverslip followed by atmospheric drying. A flow cell is fabricated around the nanospheres by gluing the coverslip on to a glass slide with two $1 \mathrm{~mm}$ holes, using a twopart epoxy. The two holes on the glass slide act as the inlet and outlet for the silver shell growth solution. The silver growth solution which is injected into the flow cell using a syringe pump is similar to the one used in ensemble experiments, except that the silver to ascorbic acid ratio was reduced to $1: 1$, in order to prevent any spontaneous silver formation in the dark. The plasmon resonance of selected individual nanoparticles is recorded using the dark-field microscope, followed by $532 \mathrm{~nm} \mathrm{cw}$ laser irradiation for varying amounts of time, to synthesize the Au@Ag core@shell nanospheres. 


\section{Instrumentation}

In situ extinction spectroscopy of Au@Ag core@shell nanorod synthesis is performed by placing a tungsten halogen light source (Ocean optics HL-2000) and a spectrometer (Ocean optics HR 4000) normal to the laser irradiation pathway. A $365 \mathrm{~nm}$ long pass filter coupled with neutral density filters is placed in front of the light source to remove any ultraviolet light which could trigger self-nucleation of silver nanoparticles. In all experiments, including in the dark, the growth of Au@Ag core@shell nanorods is followed in real time with visible light transmission spectroscopy, using a low intensity tungsten halogen light source. In order to rule out any contribution to the reaction rate of the halogen lamp, we performed a control experiment in the absence of continuous illumination and measuring the plasmon resonance at intervals of 1 hour. We did not find any difference in the optical properties of the solution when compared to the one under continuous illumination with the low intensity halogen lamp. Ex situ extinction spectroscopy is performed using a Perkin Elmer Lambda 1050 UV-Vis-NIR spectrometer. $532 \mathrm{~nm}$ laser excitation of gold nanorods is performed using a CNI-MGL-532 continuous wave laser, whose power can be varied from 0 $-470 \mathrm{~mW} .730 \mathrm{~nm}$ laser irradiation is performed using a Crystalaser DL730-050-0 cw laser, whose power can be varied from $0-55 \mathrm{~mW}$. Laser powers are measured using a thermopile sensor (Newport, 919P-030-18) which is connected to a power meter (Newport, 2936-R). The laser beam profile and diameters for ensemble experiments are measured using a CCD camera beam profiler (Thorlabs). The mean diameter of the $532 \mathrm{~nm}(240 \mathrm{~mW})$ and $730 \mathrm{~nm}$ (55 $\mathrm{mW}$ ) laser spots are $1.5 \mathrm{~mm}$ and $1.7 \mathrm{~mm}$ respectively. TEM measurements are performed by centrifuging the core@shell nanorods and then drop casting them on carbon membranes supported on a Cu grid, which is then taken to a JEOL JEM-2001 transmission electron microscope. Thermal characterizations are performed using a Fluke Ti200 infrared 
camera and a K-type thermocouple (CHAL-005) connected to a data logger (OM-ELUSB-TC), both obtained from Omega Engineering Limited. Dark-field single-particle spectroscopy is performed on a Zeiss Axio Observer microscope equipped with a scanning $x, y$ piezo-stage. The plasmon resonance of nanoparticles before and after illumination are measured by illuminating them with an LED lamp (Zeiss Illuminator microLED) focused through an EC epiplan 50x objective $(N A=0.75)$ via a dark-field reflector. The back scattered light from the nanoparticles is collected with the same objective and guided to an Andor Newton EMCCD camera coupled to an Andor Shamrock 500i spectrometer. For laser illumination experiments, a collimated Gaussian beam ( $\sim 308 \mu \mathrm{m}$ diameter) is directed on to the coverslip containing gold nanoparticles using the same objective but using a bright-field reflector cube. The laser beam (CNI laser, MGL-FN-532) power is kept at $43 \mathrm{~mW}$. The laser power at the sample is measured by keeping the Newport thermopile sensor close to the microscope objective.

\section{Optical calculations}

The absorption and scattering cross-sections, electric field enhancements, and the absorbed power inside the Au and Au@Ag core@shell nanorods are calculated using Lumerical FDTD. All simulations are performed with a background refractive index of 1.333, corresponding to water. A total-field scattered-field (TFSF) light source with a wavelength range of $400-950 \mathrm{~nm}$ is used in the simulations. For gold, we use the dielectric function from Johnson and Christy ${ }^{4}$ and for silver, we use dielectric functions from Yang et al. ${ }^{5}$ The mesh size is set to $0.25 \mathrm{~nm}$. gold nanorods corresponding to growth stage $\mathrm{NR}_{0}$ are modelled as rounded cylinders with a length of 78 nm and a width of $25 \mathrm{~nm}$. Au@Ag core@shell nanorods are modelled by replicating the structures obtained from TEM measurements. The 
growth stage $\mathrm{NR}_{1}$ is modelled with a conformal $1 \mathrm{~nm}$ silver shell around the aforementioned gold nanorod. Growth stage NR2 is modelled as a cuboidal Au@Ag core@shell nanorod, with a silver shell thickness of $3.5 \mathrm{~nm}$ on the side, $4 \mathrm{~nm}$ on the tips and a $3 \mathrm{~nm}$ radius of curvature, while growth stage $\mathrm{NR}_{3}$ is approximated using a $10 \mathrm{~nm}$ conformal silver shell.

Monte Carlo modelling of photon propagation inside the reaction vessel is performed using a Monte Carlo script in MATLAB and the heat transfer simulations are performed using COMSOL. A detailed description of these procedures have been reported previously. ${ }^{2,6}$

Monte Carlo modelling of the hot carrier propagation is performed using MATLAB. $10^{8}$ carriers are placed in a $66 \mathrm{~nm}$ sphere, with a spatial distribution proportional to the electric field intensity $|\vec{E}|^{2}$ inside the nanoparticle. Each carrier is then given a random displacement $(r, \theta, \phi)$ in spherical coordinates, where $r$ is the propagation length, $\theta$ the azimuthal angle, and $\phi$ the polar angle. The propagation length $r$ obeys an exponential probability distribution with a $1 / e$ equal to the mean free path. For interband carriers, $\theta$ follows a random distribution in which every angle is equally probable. For intraband carrier, $\theta$ follows an angular distribution $R_{\text {eff }}(\theta):^{7}$

$$
R_{e f f}(\theta) \propto a\left(\frac{3}{4} \cos ^{2} \theta+\frac{1}{4}\right)+2(1-a)\left|\cos ^{3} \theta\right|
$$

where, $a$ is the fraction of carriers generated via phonon/defect scattering. For spherical particles, $a \approx 0.45 .^{7}$ Lastly, $\phi$ obeys a random distribution in which all angles are equally probable. The carriers plotted in Figs. 4 d,e are those that have their final position outside of the nanoparticle. For simplicity, we neglect any losses due to momentum mismatch at the interface. 
Mie calculations ${ }^{8}$ of gold nanospheres and Au@Ag core@shell nanospheres in a homogenous environment are performed using a script in MATLAB based on the formalism laid by Bohren and Huffman? . 
Supplementary S1: Morphological and optical characterization of Au \& Au@Ag core@shell

\section{nanorods}

In this section, we present the morphological and optical characterizations of bare gold nanorods and the Au@Ag core@shell nanorods at different growth stages. We also correlate the spectral shifts observed during the silver shell growth experiment with their morphological changes, which is further corroborated using finite-difference time-domain (FDTD) calculations.
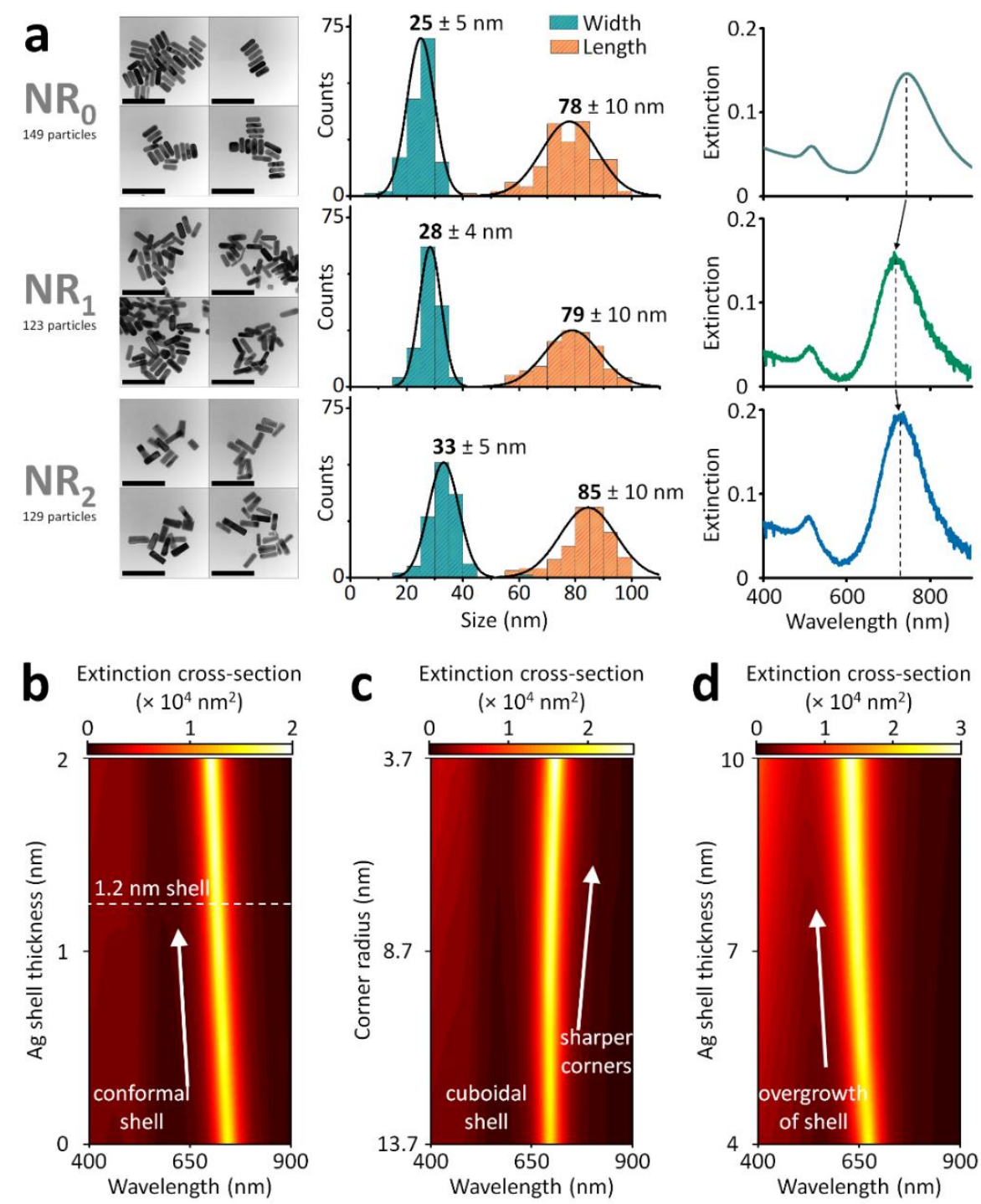

Figure S1. (a) TEM images, size distribution, and extinction spectra of bare gold nanorods ( $N R_{0}, 1^{\text {st }}$ row), gold nanorods with $1 \mathrm{~nm}$ conformal silver shell $\left(\mathrm{NR}_{1}, 2^{\text {nd }}\right.$ row), and gold 
nanorods with a $4 \mathrm{~nm}$ cuboidal silver shell $\left(\mathrm{NR}_{2}, 3^{\text {rd }}\right.$ row). The scalebar in TEM images correspond to $200 \mathrm{~nm}$. The black line is the histograms are the corresponding Gaussian fittings. (b, c, d) FDTD calculated extinction cross-sections of gold nanorods suspended in water with a dimension of $78 \times 25 \mathrm{~nm}$ and (b) a conformal silver shell with thicknesses varying from $0-2 \mathrm{~nm}$, (c) a cuboidal silver shell with corner radii varying from $3.7 \mathrm{~nm}-13.7$ $\mathrm{nm}$, and (d) a conformal silver shell with thicknesses varying from 4-10 nm.

The colloidally synthesized bare gold nanorods $\left(N R_{0}\right)$ exhibit a transverse and longitudinal resonance at $514 \mathrm{~nm}$ and $744 \mathrm{~nm}$, respectively (Figure S1a). The size distribution of the gold nanorods is calculated by measuring the length and diameter of individual nanorods in transmission electron micrographs, using ImageJ software. Similar size distributions from TEM images have been calculated for different growth stages $\mathrm{NR}_{1}$ and $\mathrm{NR}_{2}$ (Figure S1a). For the growth stage $\mathrm{NR}_{3}$, analyzing size distribution is difficult due to the anisotropic shell growth. The correlations regarding the spectral shifts in Figure $1 \mathrm{c}$ (main text) with the morphological changes is further corroborated using FDTD calculations (Figure S1b,c,d).

We first simulate the extinction of gold nanorods with a conformal silver shell around them. The silver shell thickness is varied between $0-2 \mathrm{~nm}$ in our simulations (Figure S1b). We observe an increasing blue shift of the longitudinal plasmon resonance with increasing silver shell thickness, which is consistent with the experimentally measured spectral features at the growth stage $\mathrm{NR}_{1}$ (see main text). We then simulate the extinction crosssection of cuboidal Au@Ag core@shell nanorods with varying corner sharpness representing the growth stage between $\mathrm{NR}_{1}$ and $\mathrm{NR}_{2}$. As a starting point, a $1.2 \mathrm{~nm}$ conformal silver shell is considered. We vary the corner radius from $13.7 \mathrm{~nm}-3.7 \mathrm{~nm}$ corresponding to the transition from a conformal shell to cuboidal shell. A red shift of the 
plasmon resonance is observed (Figure S1c), corroborating our correlation of the measured spectral red shifts with the TEM images at the growth stage $\mathrm{NR}_{2}$ (see main text). After growth stage $\mathrm{NR}_{2}$, a blue shift of the plasmon resonance is observed as the silver shell thickness increases, which is simulated using a conformal silver shell over a gold nanorod with shell thicknesses ranging from 4 to $10 \mathrm{~nm}$, in Figure S1d. 


\section{Supplementary S2: Modeling photon propagation in our reaction volume}

Since our nanorods display strong absorption and scattering properties at their plasmon resonances, the photon propagation inside the reaction volume can be properly calculated only by solving the complete radiative transfer equation. ${ }^{2}$ We employ a Monte Carlo model to model the photon propagation inside the nanorod solution, which has been previously described. ${ }^{2}$ Briefly, photons with a spatial distribution corresponding to the experimental laser spatial profile are injected in to the simulated reaction volume. The simulation region is meshed into cuboidal volumes. Each voxel occupies a volume of $12.5 \times 4 \times 10 \mu \mathrm{m}^{3}$. The nanorod density that we employ in our experiments is $8.2 \times 10^{9}$ nanorods $/ \mathrm{mL}$. As such, each voxel is expected to contain approximately 4 nanorods. The photons get scattered and absorbed with probabilities depending on their FDTD calculated scattering and absorption cross-sections. The photons absorbed in each mesh cell is calculated and plotted in Figure S2. Multiple photon scattering events and reflection and transmission events at the simulation boundaries are also considered in our calculations.

a

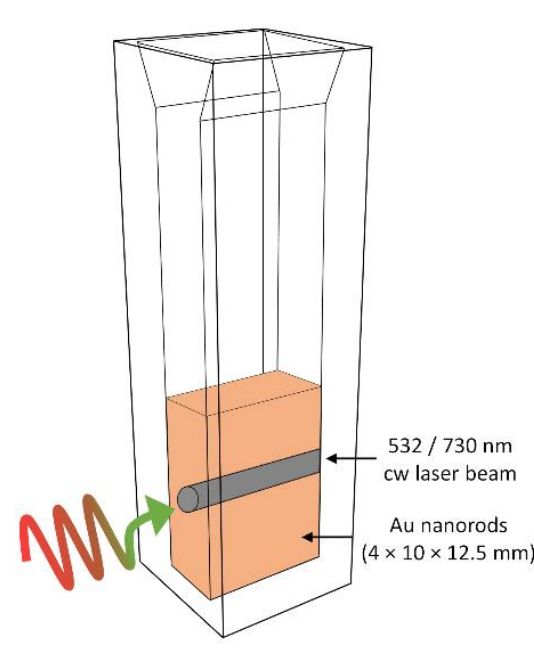

b $532 \mathrm{~nm}$ cw laser $-240 \mathrm{mw}$

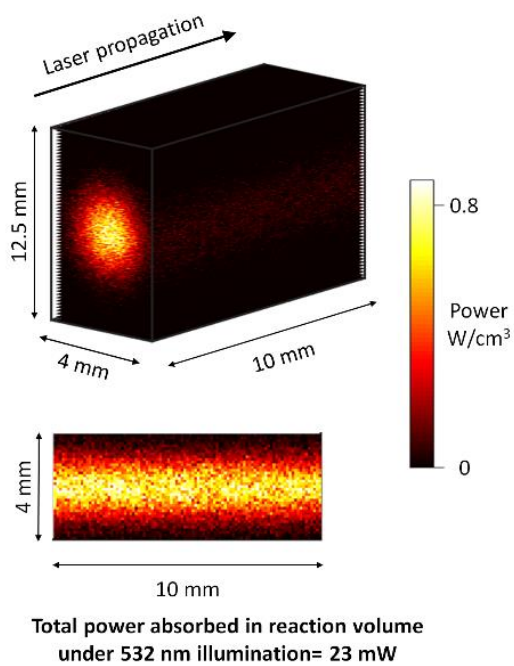

C $\quad 730 \mathrm{~nm}$ cw laser $-55 \mathrm{~mW}$

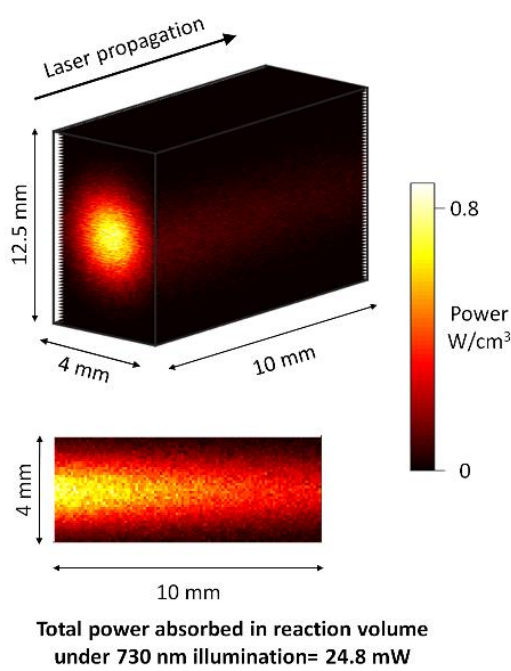

Figure S2. (a) Schematic illustration of illumination of our reaction vessel containing gold nanorods. (b, c) Calculated photon absorption inside the reaction volume under (b) $532 \mathrm{~nm}$ 
and (c) $730 \mathrm{~nm}$ laser illumination. On the bottom, heat map of calculated photon absorption at the centre of the reaction volume is displayed.

The calculated average absorption and scattering cross-sections of the nanorods at 532 $\mathrm{nm}$ illumination are $1300 \mathrm{~nm}^{2}$ and $78 \mathrm{~nm}^{2}$ respectively, while under $730 \mathrm{~nm}$ illumination the average absorption and scattering cross-sections are $7700 \mathrm{~nm}^{2}$ and $3500 \mathrm{~nm}^{2}$. Intuitively, the larger illumination power required with the $532 \mathrm{~nm}$ laser $(240 \mathrm{~mW}$ ) compared to the $730 \mathrm{~nm}$ laser ( $55 \mathrm{~mW}$ ) can be understood from the smaller absorption and scattering crosssections of the nanorods at $532 \mathrm{~nm}$ compared to $730 \mathrm{~nm}$. To ensure that the total absorbed power is the same under the two illuminations, in our Monte Carlo calculation we take into account the different absorption and scattering cross-sections, the effect of multiple scattering events, and the slightly different beam diameters of the $532(1.5 \mathrm{~mm})$ and 730 $(1.7 \mathrm{~mm})$ lasers.

Even though the spatial profile of photon absorption under $532 \mathrm{~nm}$ and $730 \mathrm{~nm}$ illumination are different, the total power absorbed inside the reaction volume, and therefore any photothermal heating, under these two conditions will be similar. 
Supplementary S3: Ex situ extinction measurements and morphological characterization under different illumination conditions at $\mathrm{t}=\mathbf{0 ~ h}$ and $24 \mathrm{~h}$.

In this section, we present ex situ extinction spectra of Au@Ag core@shell nanorods kept in the dark, under $730 \mathrm{~nm}$ illumination, and under $532 \mathrm{~nm}$ illumination at time, $\mathrm{t}=0 \mathrm{~h}$ and $\mathrm{t}$ $=24 \mathrm{~h}$. TEM images and corresponding size distributions after $24 \mathrm{~h}$ of experiment are also presented.
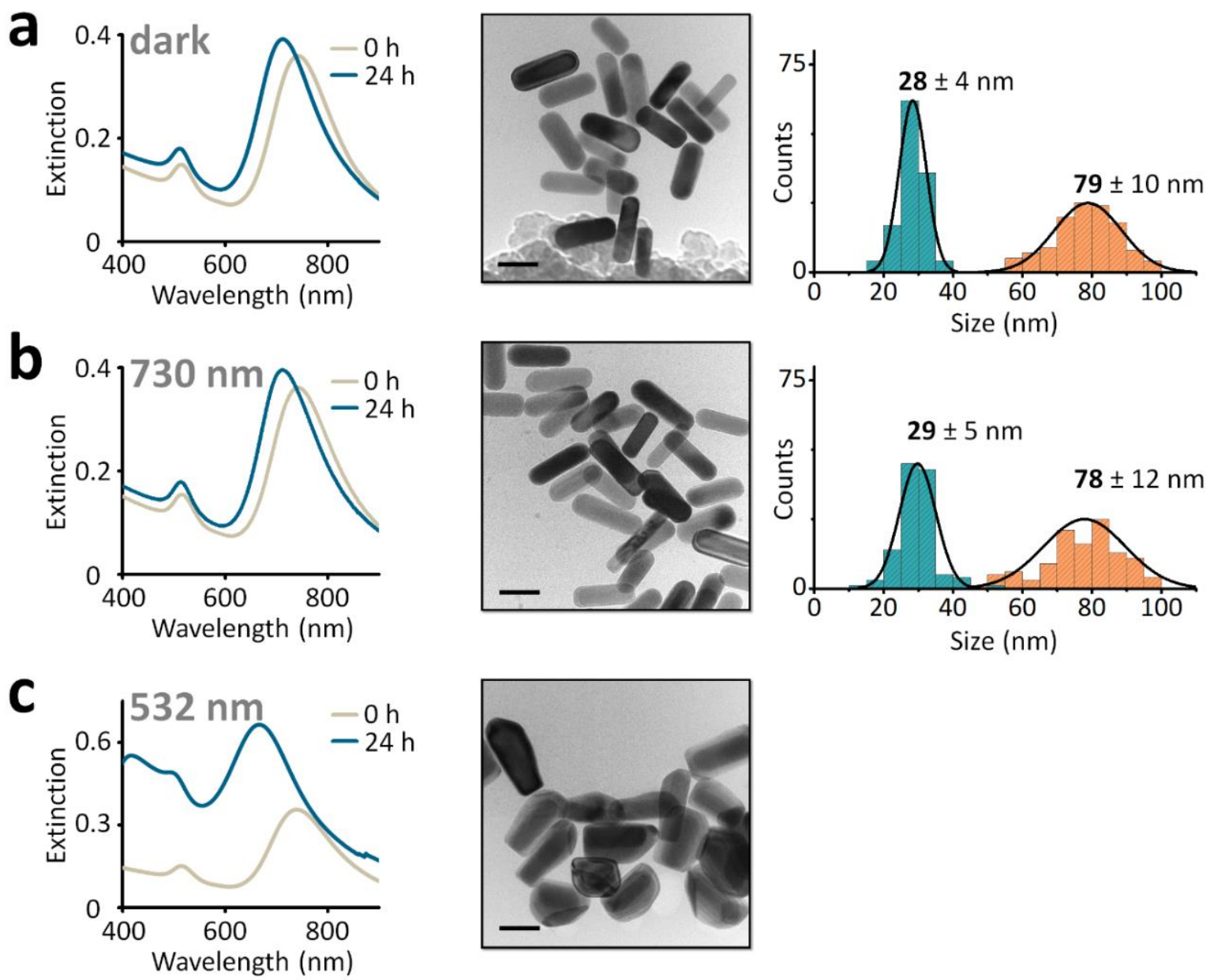

Figure S3. Extinction spectra of the Au@Ag core@shell nanorod synthesis at $\mathrm{t}=0 \mathrm{~h}$ and $\mathrm{t}=$ $24 \mathrm{~h}$ in (a) dark conditions, (b) $730 \mathrm{~nm}$, and (c) $532 \mathrm{~nm}$ laser illumination. TEM images of Au@Ag core@shell nanorods and associated size distributions measured after $24 \mathrm{~h}$ of experiment are also presented. Scalebars in the TEM images correspond to $50 \mathrm{~nm}$. The black line is the histograms are the corresponding Gaussian fittings. 
The ex situ extinction spectra of nanorod growth solutions kept in dark and under $730 \mathrm{~nm}$ illumination show similar transverse and longitudinal resonance changes (Figure S3 a,b) after $24 \mathrm{~h}$, while the solution irradiated using a $532 \mathrm{~nm}$ laser shows drastic changes (Figure S3c). The TEM images and the size distributions of Au@Ag core@shell nanorods after 24 h of synthesis under dark and $730 \mathrm{~nm}$ laser illumination look very similar, as can be observed from Figure S3a\&b. For $532 \mathrm{~nm}$ illumination, analyzing size distribution is difficult due to the anisotropic shell growth. 


\section{Supplementary S4: Evaluating plasmonic rate enhancement}

To obtain a rate enhancement from the longitudinal LSPR as a function of time (Figure $2 \mathrm{~d}$ in the main text), we first separate the time evolution in three parts: 1) start to first local minimum in the LSPR, 2) first local minimum to the next local maximum in the LSPR, 3) local maximum to the end. We remove part of the $532 \mathrm{~nm}$ illumination measurement, so that both datasets end at the same LSPR wavelength (Figure S4a). As the local minimum and maximum in the two datasets are not at the exact same wavelengths, we normalize each part of the dataset from 0 to 1 (Figure S4b). For each normalized LSPR wavelength we then find the time at which this LSPR is reached in the dark, and the time at which this LSPR is reached under $532 \mathrm{~nm}$ illumination (Figure S4c). To obtain the rate enhancement in Figure 2e of the main text, we take the derivative of the time in the dark with respect to the time under $532 \mathrm{~nm}$ illumination, i.e. the derivative of Figure $\mathrm{S} 4 \mathrm{c}$ below.
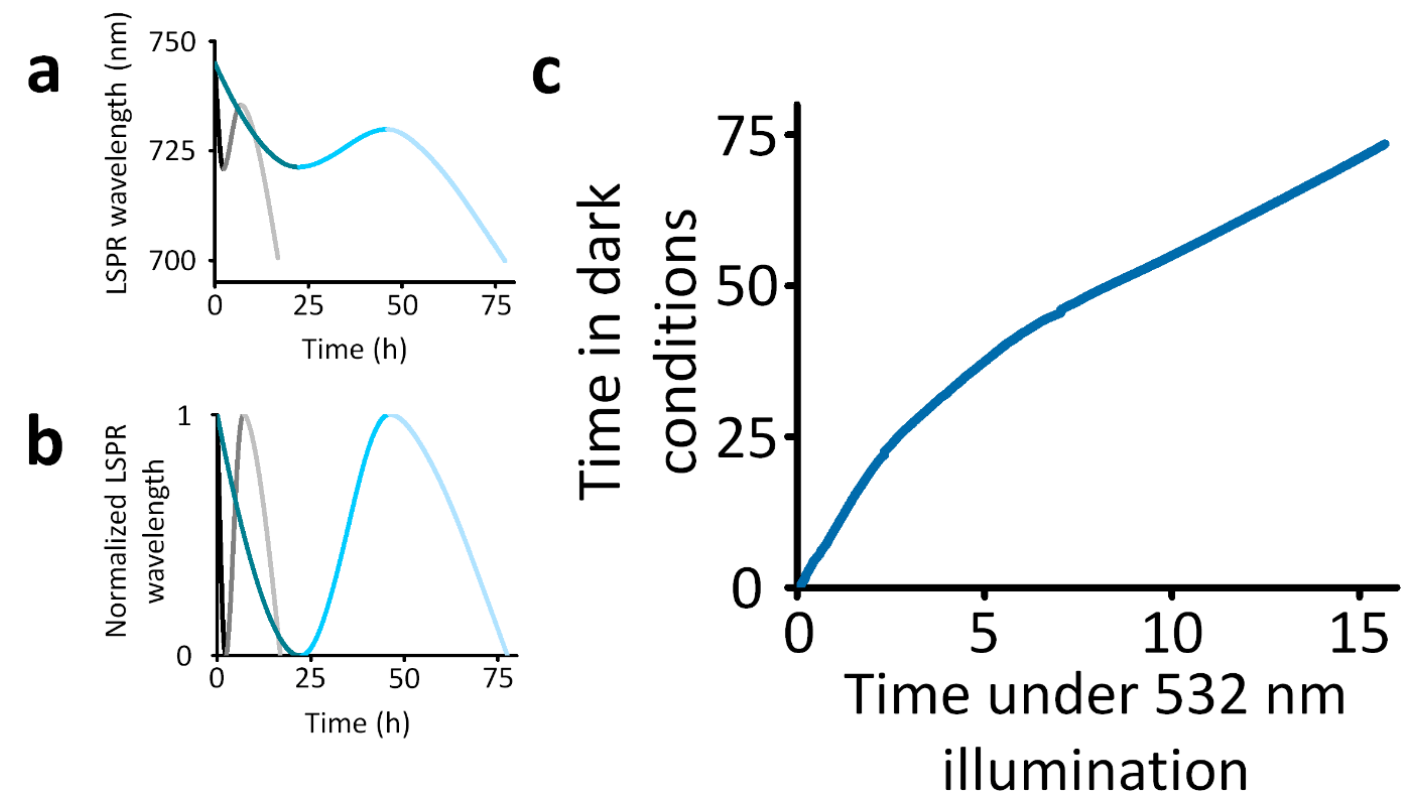

Figure S4. (a) Longitudinal LSPR as a function of time for the experiment in the dark (blue) and under $532 \mathrm{~nm}$ illumination (grey). (b) Same as panel (a), but each section is normalized from 0 to 1. (c) Time at which a certain LSPR in panel (b) is reached in the dark as a function of the time at which that same LSPR is reached in the $532 \mathrm{~nm}$ illumination experiment. 


\section{Supplementary S5: Thermal characterization under laser illumination}

Illumination of ensemble nanoparticle suspensions can generate both localized and collective photothermal heating effects.

\section{(a) Localized plasmonic heating}

Localized photothermal temperature increase on nanorods, $\Delta T$, can be calculated using (see equation 1 in main text): ${ }^{10}$

$$
\Delta T_{\text {local }}=\frac{\sigma_{a b s} I}{4 \pi \beta \kappa R_{e q}}
$$

Assuming a nanorod geometry parallel to the incident light polarization in FDTD simulations, we calculate absorption cross-sections, $\sigma_{a b s}$ of $1.32 \times 10^{-11} \mathrm{~cm}^{2}$ and $2.18 \times 10^{-10}$ $\mathrm{cm}^{2}$ at $532 \mathrm{~nm}$ and $730 \mathrm{~nm}$ wavelengths, respectively. Under our illumination geometries, the maximum power density, $I$ at the centre of the Gaussian laser beam is $13.38 \mathrm{~W} / \mathrm{cm}^{2}$ and $2.25 \mathrm{~W} / \mathrm{cm}^{2}$ for $532 \mathrm{~nm}\left(\right.$ Power $=240 \mathrm{~mW}, 1 / \mathrm{e}^{2}$ beam diameter $\left.=1.511 \mathrm{~mm}\right)$ and $730 \mathrm{~nm}$ (Power $=55 \mathrm{~mW}, 1 / \mathrm{e}^{2}$ beam diameter $=1.765 \mathrm{~mm}$ ) experiments, respectively.

The thermal capacitance coefficient, $\beta$, for a nanorod is defined as: ${ }^{10}$

$$
\beta=1+0.96587 \ln ^{2}\left(\frac{l}{w}\right)
$$

where, $l$ and $w$ are the length and width of the nanorod. We calculate a $\beta$ of 2.25 for our gold nanorods of dimension $78 \times 25 \mathrm{~nm}$.

The thermal conductivity of water, $\kappa$, is $0.6 \mathrm{~W} / \mathrm{m} / \mathrm{K}$. The equivalent radius of the nanorod, $R_{e q}$, which is calculated by equating the volume of the nanorod to that of a sphere in our case is $\sim 20 \mathrm{~nm}$.

Using the above values, we find a maximum temperature increase of 1.04 millikelvins under $532 \mathrm{~nm}$ illumination and 2.87 millikelvins under $730 \mathrm{~nm}$ illumination, on the 
nanorods. Such negligible local temperature increases cannot explain the plasmon-driven silver shell growth in our experiments.

\section{(b) Collective heating effects}

Under typical illumination geometries, multiple nanoparticles are illuminated simultaneously which leads to the generation of macroscopic collective heating effects along with heat convection. We characterize these global heating effects by measuring the temperature on the cuvette walls using an infrared camera and by measuring the temperature of the nanoparticle suspension using a thermocouple. Since thermocouples are photosensitive, in our measurements, they are placed inside the liquid suspension above the laser beam.

We also model heat dissipation inside the reaction vessel by first calculating photon absorption inside the reaction volume using Monte Carlo simulations (Supplementary S2), and then we use the calculated absorbed power as an input in COMSOL. In our COMSOL simulations, we fix the temperature at the outer boundary of the quartz cuvette at $6{ }^{\circ} \mathrm{C}$, corresponding to our set temperature in our experiments.

In the Infrared camera (IR) measurements, we set an emissivity of 0.93 corresponding to quartz to measure the temperature of the external surface of the cuvette. Infrared camera measurements in Figure S5 display temperatures of $9-10{ }^{\circ} \mathrm{C}$, despite setting the cuvette holder to $6{ }^{\circ} \mathrm{C}$. This disparity is attributed to the accuracy of $\pm 2 \mathrm{~K}$ of the IR camera, according to the website of the camera manufacturer. The IR measurements indicate a temperature increase of $\sim 0.5 \mathrm{~K}$ under illumination, on the quartz cuvette walls. In the thermocouple measurements, temperature increases of $1 \mathrm{~K}$ and $0.5 \mathrm{~K}$ are observed for 532 
$\mathrm{nm}$ and $730 \mathrm{~nm}$ illumination, respectively. The thermocouple measurements have an accuracy of $\pm 2.2 \mathrm{~K}$, according to the website of the thermocouple manufacturer.

a Infrared camera images

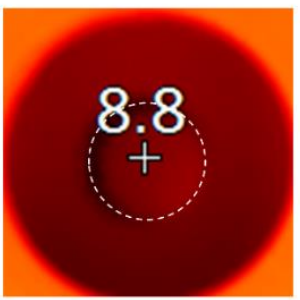

$\mathrm{t}=0 \mathrm{~min}$

C

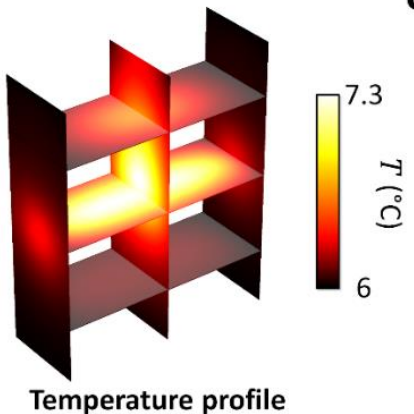

Temperature profile

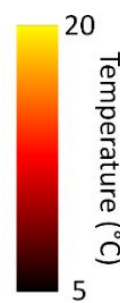

5

d

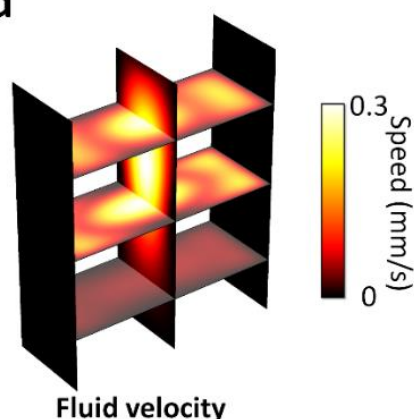

$730 \mathrm{~nm}$ cw laser $-55 \mathrm{~mW}$

f

Infrared camera images

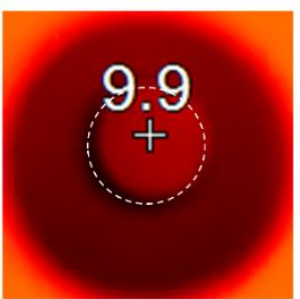

$\mathrm{t}=0 \mathrm{~min}$

h
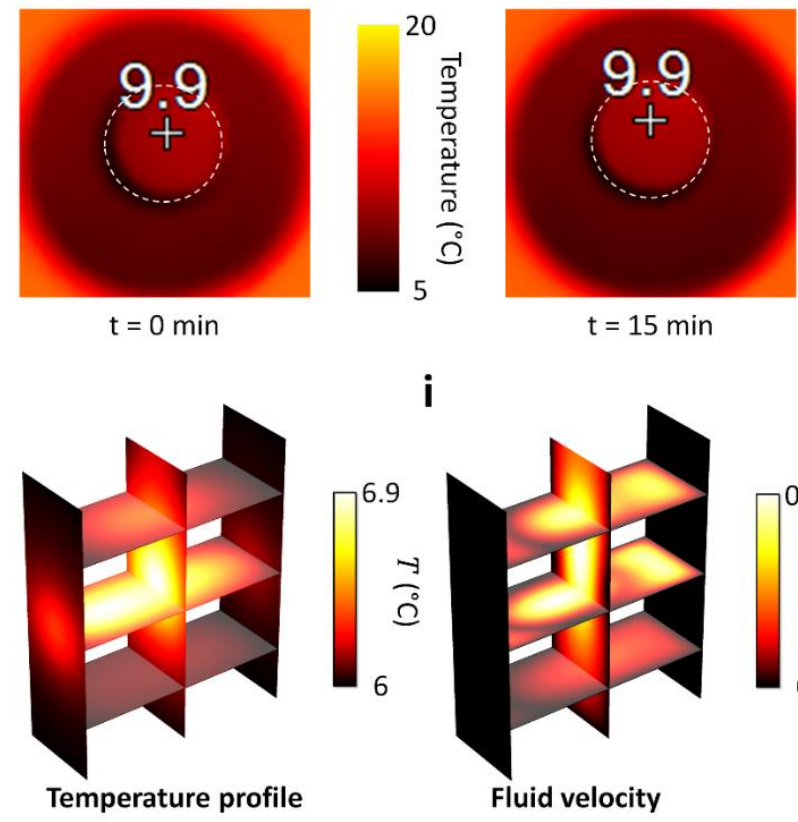

$\mathrm{t}=15 \mathrm{~min}$

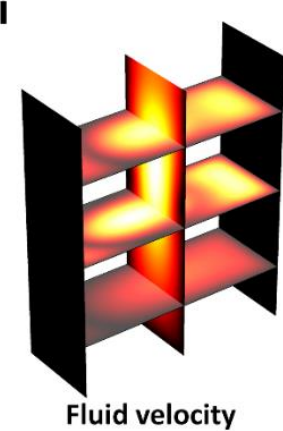

$532 \mathrm{~nm}$ cw laser $-240 \mathrm{~mW}$

b Thermocouple measurements

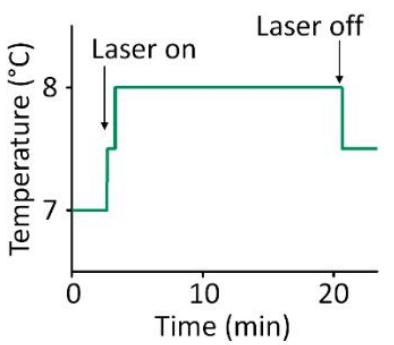

e

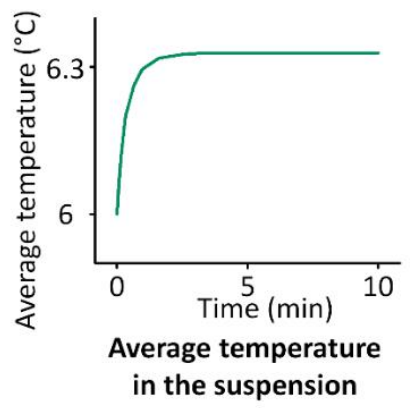

g Thermocouple measurements

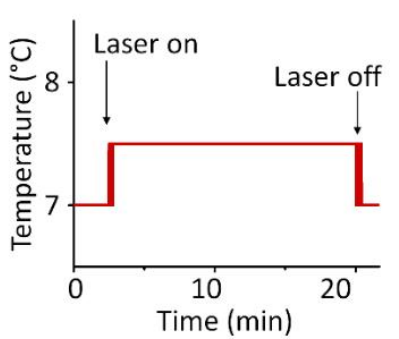

j

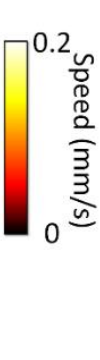

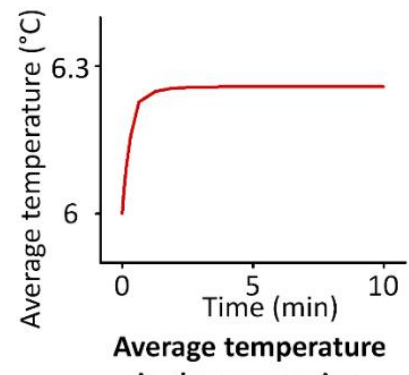

in the suspension

Figure S5. (a, b, c, d, e) (a) Infrared camera measurement at t $=0 \mathrm{~min}$ and $15 \mathrm{~min}$, (b) thermocouple temperature measurements as a function of time, COMSOL calculated (c) 
heat map of temperature profile, (d) heat map of fluid velocity and (e) evolution of average temperature of the nanorod suspensions under $240 \mathrm{~mW} 532 \mathrm{~nm}$ cW illumination. (f, $\mathbf{g}, \mathbf{h}, \mathbf{i}, \mathbf{j})$ Similar thermal characterization for $55 \mathrm{~mW} 730 \mathrm{~nm} \mathrm{cw}$ illumination. The white dashed lines in the infrared camera images denote the optical window of the cuvette holder, where the quartz cuvette with the gold nanorod suspension is held.

The thermal characterizations shown in Figure S5 indicate similar temperature increases of $\sim 1 \mathrm{~K}$ for the nanoparticle suspension under $532 \mathrm{~nm}$ and $730 \mathrm{~nm}$ illumination. Since the magnitude of photothermal effects is similar under green and red laser illumination experiments, one would expect similar silver shell growth kinetics, if the synthesis was driven by photothermal heating. As we observe silver shell growth only under $532 \mathrm{~nm}$ irradiation experiments, we can rule out the role of photothermal heating.

After calculating the photon absorption inside the reaction volume using Monte Carlo simulations (Supplementary S2), we use the calculated absorbed power as an input in COMSOL to model heat dissipation inside the reaction vessel. In our COMSOL simulations, we fix the temperature at the outer boundary of the quartz cuvette at $6{ }^{\circ} \mathrm{C}$, corresponding to our set temperature in our experiments. From the COMSOL simulations, we predict a similar average temperature increase inside the reaction volume under $532 \mathrm{~nm}$ and $730 \mathrm{~nm}$ illumination of $\sim 0.3 \mathrm{~K}$. The temperature increases calculated here are consistent with the thermocouple and infrared camera measurements we performed in the illuminated reaction vessel. Such small temperature increases cannot explain the silver shell growth rate enhancement we observe under $532 \mathrm{~nm}$ illumination. 


\section{Supplementary S6: Quantifying near-field enhancements}

We simulate the electric field enhancements at the vicinity of the nanorod surface at different growth stages, using FDTD. In Figure S6, we plot the average near-field enhancements by integrating the electric fields at various distances from 0.5 to 2,5 , and 10 $\mathrm{nm}$ from the nanorod surface.

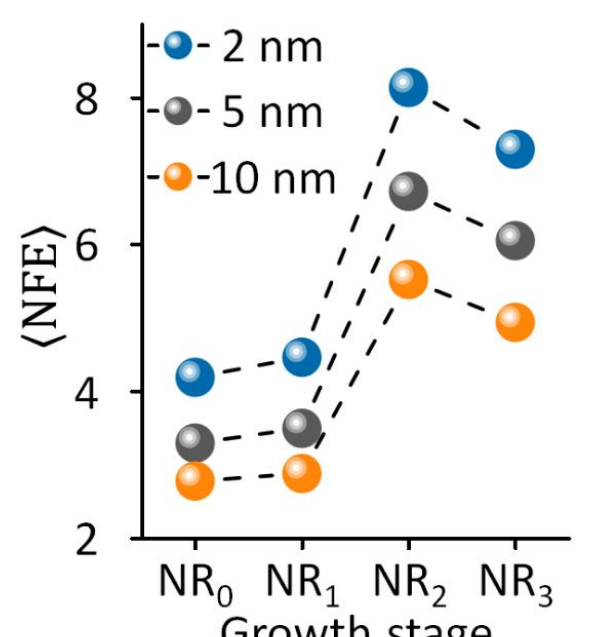

Figure S6. Average near-field enhancements around the nanorod surface plotted as a function of the silver shell growth stage. The fields are integrated at distances from $0.5 \mathrm{~nm}$ to $2 \mathrm{~nm}$ (blue), $5 \mathrm{~nm}$ (gray), and $10 \mathrm{~nm}$ (orange) from the nanorod surface.

In our simulations we observe artifacts in the electric field at the nanorod surface, due the roundness of the nanorod tips, resulting in certain mesh cells containing both the metal and the surrounding medium. To avoid these artifacts, we integrate the $N F E$ from $0.5 \mathrm{~nm}$ from the surface to various distances away from the surface (Figure S6), since the fields will decay homogeneously away from the surface. In all these cases, we observe a similar trend where the $\langle N F E\rangle$ increases with silver shell growth, while experimentally we observe a decreasing plasmonic rate enhancement (Figure $2 \mathrm{~d}$,e in main text), safely allowing us to rule out their contributions. 


\section{Supplementary S7: Temperature calculations in single-particle studies}

In this section, we demonstrate that temperature effects are negligible in our singleparticle studies.

\section{(a) Localized plasmonic heating}

To calculate the localized temperature increases under illumination in our single-particle studies, we use the following equation: ${ }^{11}$

$$
\Delta T_{\text {local }}=\frac{\sigma_{a b s} I}{4 \pi \kappa R}
$$

where, the absorption cross-section, $\sigma_{a b s}$, of our $66 \mathrm{~nm}$ gold nanospheres is $1.371 \times 10^{-14}$ $\mathrm{m}^{2}$ and the laser intensity, $I$, is kept at $165 \times 10^{4} \mathrm{~W} / \mathrm{m}^{2}$. Since the gold nanospheres lie at the interface of glass $\left(\kappa_{\text {glass }}=1 \mathrm{~W} / \mathrm{m} / \mathrm{K}\right)$ and water $\left(\kappa_{\text {water }}=0.6 \mathrm{~W} / \mathrm{m} / \mathrm{K}\right)$, an average thermal conductivity, $\kappa$, of $0.8 \mathrm{~W} / \mathrm{m} / \mathrm{K}$ is chosen for the calculations. The radius of the nanospheres is $33 \mathrm{~nm}$.

Using the above values, we find a negligible local temperature increase of 62 millikelvins on the nanoparticle that is located at the centre of the Gaussian beam. Such low heating effects are not enough to grow a silver shell around the gold spheres.

\section{(b) Collective heating effects}

To calculate the temperature increases under collective heating effects, we use the following equation that has been previously used for two-dimensional nanoparticle arrays: ${ }^{12}$

$$
\Delta T_{\text {collective }}=\frac{\sigma_{a b s} P}{\kappa} \sqrt{\frac{\ln 2}{4 \pi}} \frac{1}{H A}\left(1-\frac{4 \sqrt{\ln (2) A}}{\pi H}\right)
$$

where, $P$ is the power of illumination, $H$ is the full width at half maximum (FWHM) of the Gaussian laser beam, and $A$ is the unit cell area of the nanoparticle lattice. 
Although our nanospheres are dispersed randomly on a glass substrate (Figure S7), in order to numerically estimate the temperature increases, we assume that these particles are arranged in a square lattice in order to use the above equation. From the dark-field images of the nanoparticles, we observe that the average interparticle distance is larger than $10 \mu \mathrm{m}$. Even in the case of the closest spaced nanoparticles, we measure at least an interparticle distance of $3 \mu \mathrm{m}$ (Figure S7). Assuming an interparticle spacing of $3 \mu \mathrm{m}$ (therefore $A$ becomes $9 \mu \mathrm{m}^{2}$ ), and using experimentally measured $P$ and $H$ of the laser beam of $43 \mathrm{~mW}$ and $153.9 \mu \mathrm{m}$ respectively, we calculate a negligible temperature increase of $0.12 \mathrm{~K}$.

In reality, the average interparticle spacing is much higher than $3 \mu \mathrm{m}$, so therefore, we expect even lower temperature increases due to collective heating effects.

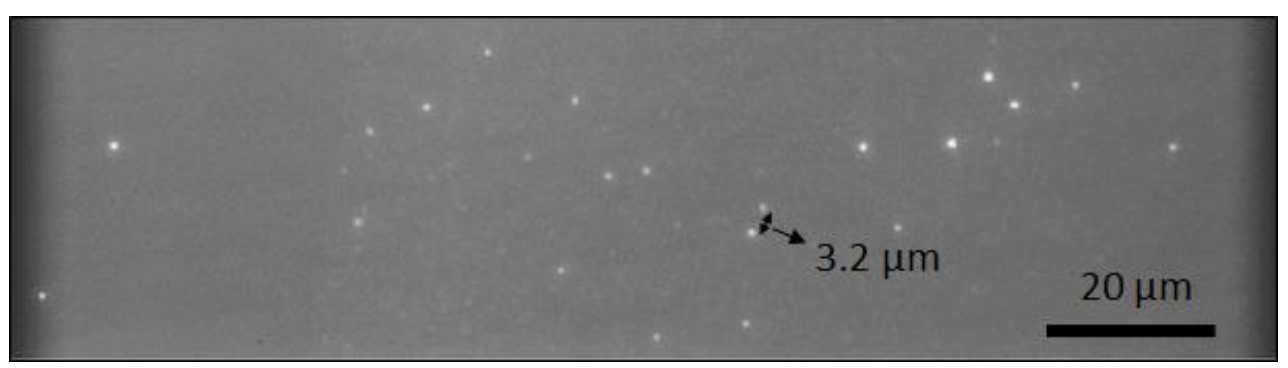

Figure S7. Dark-field scattering of gold nanoparticles dispersed on a glass substrate. 
Supplementary S8: Mie theory calculations for the growth of Au@Ag core@shell nanospheres

We calculate the scattering cross-section of Au@Ag core@shell nanospheres using Mie theory, ${ }^{8,9}$ to estimate the silver shell thickness in our single-particle studies. In our calculations, we use dielectric functions for gold and silver reported by Johnson \& Christy and Yang et al, respectively. ${ }^{4,5}$ We model a conformal silver shell with thickness from $0-5$ $\mathrm{nm}$, over a $66 \mathrm{~nm}$ gold nanospheres.
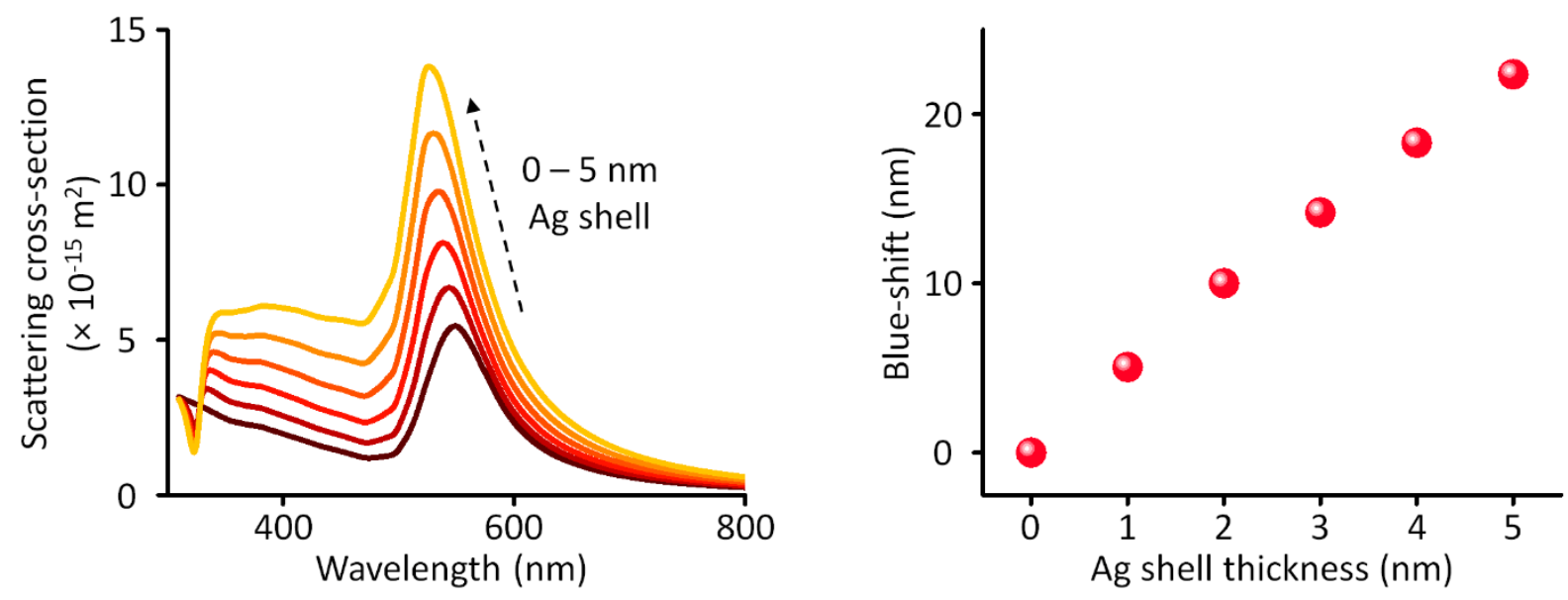

Figure S8. (left) Scattering cross-section of Au@Ag core@shell nanospheres, where the silver shell thickness is varied between 0 to $5 \mathrm{~nm}$. (right) From the data on the left plot, the blue shift of plasmon resonance is plotted as a function of silver shell thickness.

From these Mie calculations, we observe increasing blue shift of the plasmon resonance with increasing shell thicknesses. From Figure S8, for a plasmon resonance blue shift of 17 $\mathrm{nm}$ observed in Figure $5 \mathrm{~b}$ (main text), we can estimate the growth of a $4 \mathrm{~nm}$ silver shell over the gold nanosphere. 
Supplementary S9: Control experiments to confirm the plasmonic nature of silver shell growth

Several control experiments for the dark-field single-particle studies were performed to confirm the plasmonic nature of the core@shell nanosphere growth.
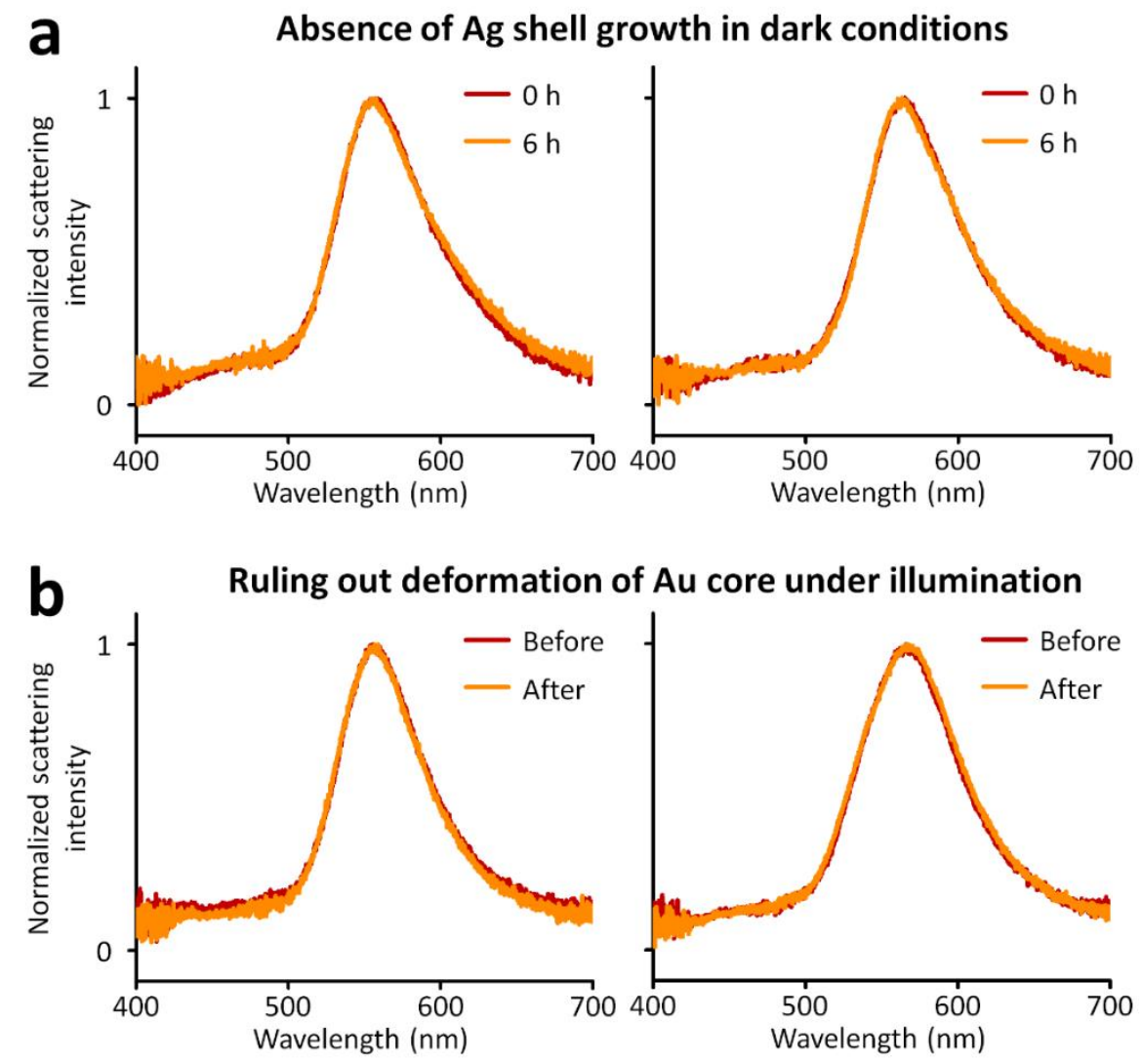

C Absence of $\mathrm{Ag}$ nucleation in the absence of Au nanoparticles
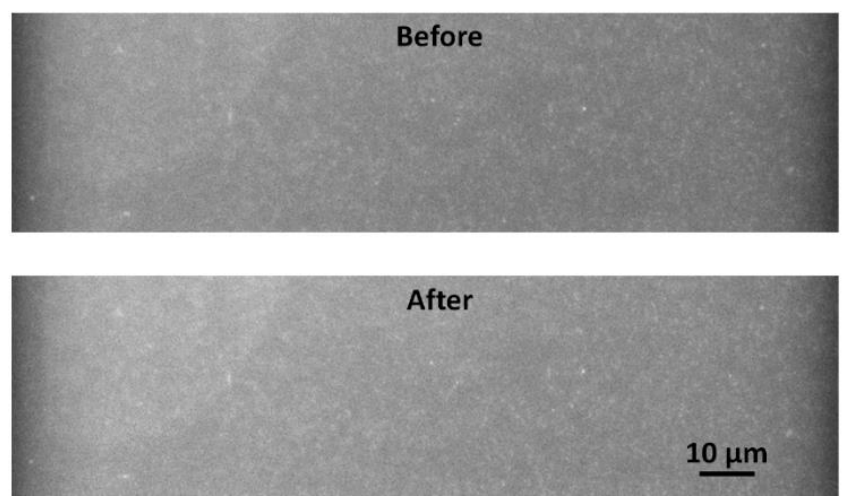

Figure S9. (a) Normalized scattering spectra of two different single gold nanoparticles at time $t=0 \mathrm{~h}$ and $t=6 \mathrm{~h}$, when kept in the dark while flowing silver growth solution. (b) Normalized scattering spectra of two different single gold nanoparticles before and after 
laser illumination for a period of $2 \mathrm{~h}$, in the absence of $\mathrm{Ag}+$ ions in the growth solution. (c) Dark-field image of the quartz cover slip before and after $532 \mathrm{~nm}$ illumination for $2 \mathrm{~h}$, while flowing the silver growth solution.

Single-particle experiments were performed in the absence of laser irradiation to characterize the silver shell growth under dark conditions (Figure S9a). In these experiments, the plasmon resonance of the gold nanoparticles is measured at the beginning and at the end of the experiment, while flowing the silver shell growth solution. No significant silver shell growth is observed under dark conditions even after $6 \mathrm{~h}$, as observed from the similar scattering spectra at the beginning and at the end of the experiment. Such control experiments show that plasmon excitation is necessary for the silver shell growth under our reaction conditions.

In order to rule out the possibility of any gold nanoparticle deformation under illumination, we irradiate the nanoparticles with a $532 \mathrm{~nm}$ laser (Figure S9b). A solution consisting of BSPP and ascorbic acid is injected in to the flow cell, but in the absence of any $\mathrm{Ag}^{+}$ions. Under illumination, the gold nanoparticles do not show any spectral change as observed in Figure S9b, thus allowing us to rule out any nanoparticle deformation under laser irradiation.

In order to verify if any silver nucleation occurs in the absence of gold nanospheres, we fabricate a flow cell without depositing any gold nanoparticles on the coverslip. We flow the silver growth solution through the flow cell and simultaneously illuminate it with a $532 \mathrm{~nm}$ cw laser. From Figure S9c, we do not observe the formation of any bright scatterers after illumination, suggesting that no large silver nanoparticles are formed on the surface. 


\section{Supplementary S10: Monte Carlo simulations of hot carrier propagation}

We first simulate the power absorbed $P_{a b s}$ at $532 \mathrm{~nm}$ in a $66 \mathrm{~nm}$ gold sphere (Figure S10a). $10^{8}$ carriers are then placed in the sphere with a spatial distribution proportional to $P_{a b s}$, which is proportional to $|\vec{E}|^{2}: P_{a b s}=\frac{1}{2} \omega|\vec{E}|^{2} \operatorname{Im}[\varepsilon]$, where $\omega$ is the photon frequency and $\operatorname{Im}[\varepsilon]$ the imaginary part of the dielectric function $\varepsilon$. Each carrier is given a random displacement $(r, \theta, \phi)$ in spherical coordinates, where $r$ is the propagation length, $\theta$ the azimuthal angle, and $\phi$ the polar angle. The propagation length $r$ obeys an exponential probability distribution with a $1 / e$ equal to the mean free path. These random numbers for $r$ are easily generated using inverse transform sampling.

For interband carriers, $\theta$ follows a random distribution in which every angle is equally probable. For intraband carriers, $\theta$ follows an angular distribution $R_{e f f}(\theta):^{7}$

$$
R_{e f f}(\theta) \propto a\left(\frac{3}{4} \cos ^{2} \theta+\frac{1}{4}\right)+2(1-a)\left|\cos ^{3} \theta\right|
$$

where, $a$ is the fraction of carriers generated via phonon/defect scattering. For spherical particles, $a \approx 0.45 .{ }^{7}$ Generating random numbers from this angular distribution is not trivial, as the inverse cumulative distribution function of $R_{e f f}(\theta)$ cannot be calculated analytically. Instead, we approximate $R_{\text {eff }}(\theta)$ with a superposition of Gaussian distributions centered at $0, \pi, 2 \pi$, and a width $\sigma$ of 0.6 radians (Figure S10b).

Lastly, $\phi$ obeys a random distribution in which all angles are equally probable. The carriers plotted in Figure $5 \mathrm{~d}$,e of the main text are those that have their final position outside of the nanoparticle. For simplicity, we neglect any losses due to momentum mismatch at the interface, so the carriers plotted in Figure $5 \mathrm{~d}$,e of the main text can also be interpreted as those that reach the nanoparticle surface. 

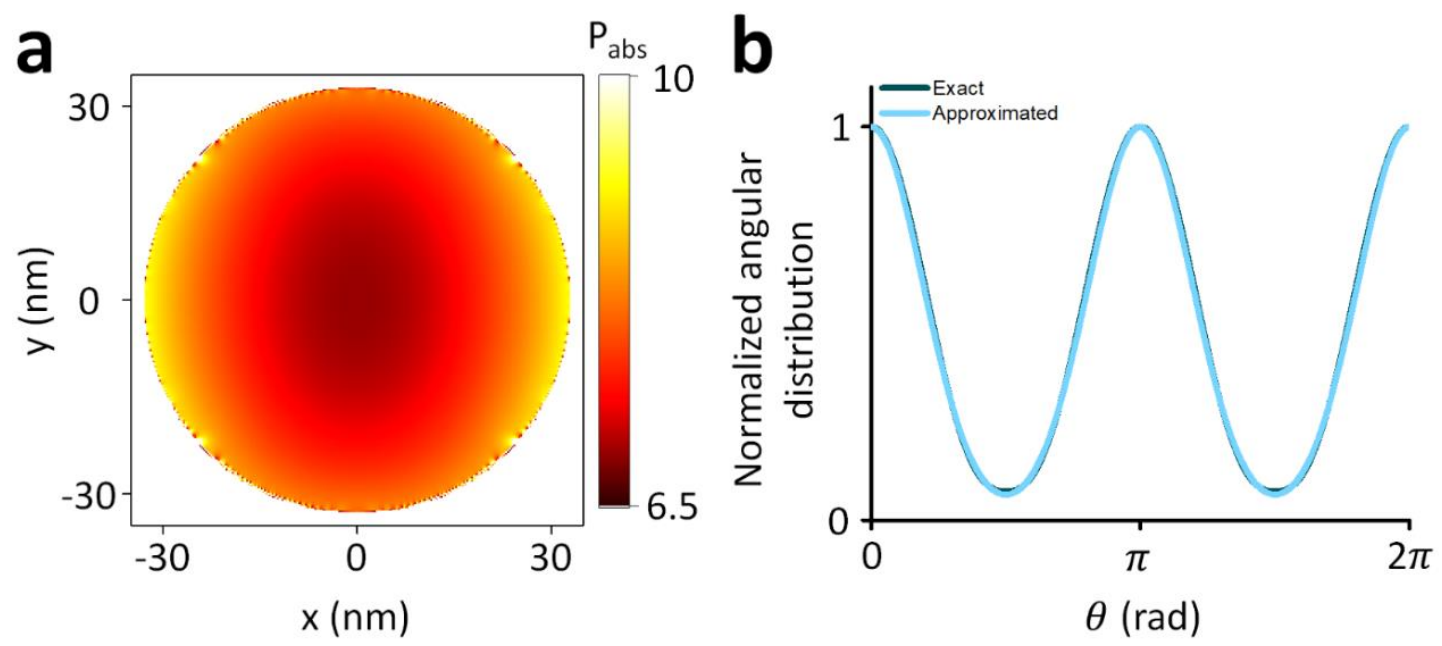

Figure S10. (a) Two-dimensional slice of the absorbed power at the center of the nanoparticle. The units for $P_{a b s}$ are normalized and can be converted to $\mathrm{W} / \mathrm{m}^{3}$ by multiplying with the power that is experimentally injected on an area equivalent to the simulated source area. The polarization is along the $y$ axis. (b) Normalized angular distribution $R_{\text {eff }}$ (labeled 'exact') and the approximation used in the simulation (labeled 'approximated'). 


\section{References}

(1) Ye, X.; Jin, L.; Caglayan, H.; Chen, J.; Xing, G.; Zheng, C.; Doan-Nguyen, V.; Kang, Y.; Engheta, N.; Kagan, C. R.; et al. Improved Size-Tunable Synthesis of Monodisperse Gold Nanorods through the Use of Aromatic Additives. ACS Nano 2012, 6 (3), 2804-2817. https://doi.org/10.1021/nn300315j.

(2) Kamarudheen, R.; Castellanos, G. W.; Kamp, L. P. J.; Clercx, H. J. H.; Baldi, A. Quantifying Photothermal and Hot Charge Carrier Effects in Plasmon-Driven Nanoparticle Syntheses. ACS Nano 2018, 12 (8), 8447-8455. https://doi.org/10.1021/acsnano.8b03929.

(3) Zheng, Y.; Zhong, X.; Li, Z.; Xia, Y. Successive, Seed-Mediated Growth for the Synthesis of Single-Crystal Gold Nanospheres with Uniform Diameters Controlled in the Range of 5-150 Nm. Part. Part. Syst. Charact. 2014, $31 \quad$ (2), 266-273. https://doi.org/10.1002/ppsc.201300256.

(4) Johnson, P. B.; Christy, R. W. Optical Constants of the Noble Metals. Phys. Rev. B 1972, 6 (12), 4370-4379.

(5) Yang, H. U.; D’Archangel, J.; Sundheimer, M. L.; Tucker, E.; Boreman, G. D.; Raschke, M. B. Optical Dielectric Function of Silver. Phys. Rev. B 2015, 91 (23), 235137. https://doi.org/10.1103/PhysRevB.91.235137.

(6) Hogan, N. J.; Urban, A. S.; Ayala-Orozco, C.; Pimpinelli, A.; Nordlander, P.; Halas, N. J. Nanoparticles Heat through Light Localization. Nano Lett. 2014, 14 (8), 4640-4645. https://doi.org/10.1021/nl5016975.

(7) Khurgin, J. B. Fundamental Limits of Hot Carrier Injection from Metal in Nanoplasmonics. Nanophotonics 2019.

(8) Mie, G. Beiträge Zur Optik Trüber Medien, Speziell Kolloidaler Metallösungen. Ann. Phys. 1908, 330 (3), 377-445. https://doi.org/10.1002/andp.19083300302.

(9) Bohren, C. F. \& Huffman, D. R. Absorption and Scattering of Light by Small Particles. WILEYVCH Verlag. WILEY-VCH Verlag April 23, 1998, pp 57-81. 
(10) Baffou, G.; Quidant, R.; Garcia de Abajo, F. J. Nanoscale Control of Optical Heating in Complex Plasmonic Systems. ACS Nano 2010, 4, 709-716.

(11) Baffou, G.; Rigneault, H. Femtosecond-Pulsed Optical Heating of Gold Nanoparticles. Phys. Rev. B 2011, 84 (3), 35415.

(12) Baffou, G.; Berto, P.; Bermudez Urena, E.; Quidant, R.; Monneret, S.; Polleux, J.; Rigneault, H. Photoinduced Heating of Nanoparticle Arrays. ACS Nano 2013, 7 (8), 6478-6488. https://doi.org/10.1021/nn401924n. 